\title{
Cidofovir Efficacy in Recurrent Respiratory Papillomatosis: A Randomized, Double-Blind, Placebo-Controlled Study
}

\author{
J. Scott McMurray, MD; Nadine Connor, PhD; Charles N. Ford, MD
}

\begin{abstract}
Objectives: We performed a prospective, double-blind, placebo-controlled, longitudinal adjuvant therapy trial to determine the efficacy of cidofovir in the treatment of severe recurrent respiratory papillomatosis (RRP). Although results of case series suggest that cidofovir may decrease the frequency and rapidity of papilloma regrowth, no blinded placebocontrolled studies have demonstrated efficacy.

Methods: Adults and children $(\mathrm{n}=19)$ with aggressive RRP received either active drug (cidofovir) or placebo. When surgical intervention was needed, drug or placebo was injected into affected areas after surgical removal of disease. The following measures were made at baseline and at 2-month intervals for the course of 12 months: Derkay papilloma severity grading scale, Voice Handicap Index, Health-Related Quality of Life, and total number of procedures performed over 12 months.

Results: At 2- and 12-month follow-ups, there was a significant $(\mathrm{p}<.05)$ improvement in the Derkay Severity Score within the cidofovir and placebo groups, but no difference between groups, and no difference between groups in the number of procedures performed. Significant improvement was found in Voice Handicap Index scores in the cidofovir group at the 12-month follow-up. No differences were seen in Health-Related Quality of Life.

Conclusions: A randomized, blinded, placebo-controlled trial is necessary in the study of RRP, because the natural history of the disease can include remissions and reactivations. We found a significant improvement in the Derkay Severity Score 12 months after the baseline assessment in patients treated with cidofovir. This effect, however, was also seen in the placebo group. Accordingly, we were unable to provide proof of efficacy of cidofovir in the treatment of RRP.
\end{abstract}

Key Words: cidofovir, recurrent respiratory papillomatosis.

\section{INTRODUCTION}

The human papillomavirus (HPV) has been found to cause recurrent respiratory papillomatosis (RRP) in the aerodigestive tract in both children and adults. The natural history of the disease is typified by spontaneous remission and reactivation, which increase the difficulty in studying the efficacy of treatment. Although the disease is rarely fatal, its management often includes prolonged and extensive medical and surgical treatment that can cause physical and emotional suffering for the patient and family. The potential risk of iatrogenic complications increases with the number of surgical interventions required to maintain an adequate airway; therefore, adjuvant therapies to decrease or arrest the growth of the recurrent respiratory papilloma would be very desirable. Although enthusiasm continues for many adjuvant therapies, none have been studied by a controlled and blinded approach with appropriate randomization of participants. Thus, we sought to investigate the efficacy of adjuvant intralesional cidofovir in a randomized, placebo-controlled, and double-blinded manner. We hypothesized that use of cidofovir would decrease the Derkay Severity Scores in our surgical patients with RRP.

Three clinical patterns of RRP exist: juvenileonset, adult-onset, and juvenile-onset with persistence into adulthood. ${ }^{1}$ As stated by Naiman et al, ${ }^{2}$ the presentations in adults and children have mild differences in clinical expression. Naiman et $\mathrm{al}^{2}$ also confirmed that although RRP is a disease of childhood, the onset of RRP can occur at any age. The aggressive form, more common in children, can also occur in adults. The clinical course, they added, is often unpredictable. ${ }^{3}$ The incidence of the juvenile form of RRP has been estimated to be around 4.3 per 100,000 children $(0.0043 \%)$ in the United States. ${ }^{4}$ The worldwide incidence of HPV infection of the cervix without atypia ranges from $1.4 \%$ in Spain to $25 \%$ in Nigeria. ${ }^{5}$ Human papillomavirus was also

From the Division of Otolaryngology-Head and Neck Surgery, Department of Surgery, University of Wisconsin School of Medicine and Public Health, Madison, Wisconsin.

Presented at the meeting of the American Broncho-Esophagological Association, San Diego, California, April 26-27, 2007. Recipient of the Broyles-Maloney Award.

Correspondence: J. Scott McMurray, MD, Otolaryngology-Head and Neck Surgery, University of Wisconsin School of Medicine and Public Health, 600 Highland Ave, K4-710 CSC, Madison, WI 53792. 
found in $2 \%$ of tonsil specimens taken from patients without a history of RRP. ${ }^{6}$ The relatively high prevalence of HPV compared to the relatively low occurrence of RRP, as well as the differences in clinical presentations, suggests that there are unidentified host factors that determine disease expression.

Presently, surgical resection of bulky disease, either by various lasers or by powered microresector, ${ }^{7,8}$ is the gold standard for managing RRP. The maintenance of a patent airway while preserving voice and swallowing is of utmost concern. Any adjuvant found to decrease the recurrence or to induce remission would be welcomed.

Currently, there are no available antiviral agents specific for HPV. Various adjuvant therapies have been attempted, including radium implants, radiotherapy, chemical or electrical fulguration, destruction with escharotics, and the application of calcined magnesium. Vaccines not specific for HPV have been tried, including bovine wart vaccine, smallpox vaccine, and mumps vaccine. Immune modulation has also been attempted with alpha-interferon, methotrexate, cimetidine, ribavirin, indole-3-carbinol, and 13-cis-retinoic acid. Furthermore, antivirals with higher affinity for viral particles other than HPV have also been used, including acyclovir and cidofovir. $^{1}$

Cidofovir has gained much enthusiasm in the treatment of RRP. It is approved for the treatment of cytomegalovirus (CMV) retinopathy in patients with acquired immunodeficiency syndrome (AIDS). Cidofovir acts as a cytosine nucleotide analog and suppresses DNA replication, with a higher affinity against viral DNA synthesis. It has the highest affinity for CMV, herpesvirus (HSV), and adenovirus DNA synthesis, but also has properties against HPV. Cidofovir may be administered topically, systemically, or by local injection into lesions.

Many case series involving intralesional cidofovir have been reported with various but encouraging results. ${ }^{9-15}$ Although the concentration and frequency of cidofovir administration differed among the case series, the results generally demonstrated that the majority of patients had a complete or partial remission during the study period. None of the case studies, however, included a control or were blinded to the treatment regimen.

Because the natural history of RRP includes spontaneous remission or reactivation, the purpose of our study was to investigate the efficacy of adjuvant intralesional cidofovir administration during the surgical treatment of RRP in a blinded, placebo-controlled manner.

\section{METHODS}

Participants. The participants in this randomized, double-blind, placebo-controlled clinical trial were 19 consecutive adult or pediatric patients (5 to 17 years of age) who presented to the University of Wisconsin Otolaryngology-Head and Neck Surgery Clinic with a diagnosis of RRP who met inclusion/exclusion criteria and gave informed consent to participate. Minors also provided assent to participate. This study was approved by the University of Wisconsin Health Sciences Institutional Review Board and received a "safe to proceed" designation from the US Food and Drug Administration (FDA; Investigational New Drug 58,481) for the off-label research use of cidofovir. The FDA dictated the drug concentrations allowed for use in this study.

To be considered for inclusion, the patients must have had 4 prior surgeries or other treatment for RRP within the past calendar year. These treatments were most often surgical vaporization or debridement, but could have also included trials of immunomodulating agents, chemotherapeutic agents, antiviral agents, hormonal agents, or antireflux medications. Accordingly, the participants manifested severe RRP for which other avenues of treatment had been exhausted. The exclusion criteria were pregnancy or plan for pregnancy within 12 months after admission to the study; breastfeeding; renal insufficiency or history of renal disease; use of nephrotoxic medications if it was not possible to discontinue their use before study participation; use of systemic or topical medications for HPV, including all types of chemotherapeutic agents and adjuvant therapy such as interferon or steroids; history of malignancies or carcinoma of any type; immune deficiency; radiotherapy of the larynx, trachea, bronchi, or lungs; and baseline laryngeal biopsies that indicated the presence of carcinoma of any type, including verrucous carcinoma. In addition, patients were excluded if they had a hypersensitivity to probenecid or if laboratory findings were abnormal, including creatinine levels and clearance, proteinuria, blood urea nitrogen, hemocrit, white blood cell and platelet counts, liver function tests, alkaline phosphatase, serum glutamic-oxaloacetic transaminase, L-lactate dehydrogenase, amylase, and creatine phosphokinase.

Study Design and Procedures. The participants were randomly assigned to either a cidofovir injection group or a placebo group according to a predetermined random schedule stratified for adults versus children. A double-blind, placebo-controlled experimental design was used. The placebo treatment was injection of saline solution that had a color and 
viscosity identical to those of the active drug. The University of Wisconsin Pharmaceutical Research Center managed participant randomization and preparation of the drug and placebo. Gilead Sciences (Foster City, California) donated study medication to the investigation.

The study participation lasted for 12 months. At the first study visit, the participant's upper aerodigestive tract was examined with videoendoscopy and baseline RRP severity was quantified with a published RRP staging instrument, the Derkay Severity Score. ${ }^{16}$ The Derkay Severity Score has a range from 0 to 86 . A lower Derkay Severity Score indicates less severe disease and an improved clinical course for RRP. In addition, as part of a larger protocol, health-related quality of life was assessed via the Quality Metric Short Form 12 (SF-12) for adults ${ }^{17}$ and the Pediatric Quality of Life Inventory (PedsQL) for children. ${ }^{18}$ After the baseline evaluation, the participants were seen in the clinic at the end of five 2-month time intervals over the course of 1 year, for a total of 6 visits for the recording of study data. Assessments were made at these time points regardless of the scheduling of surgical interventions.

The participants received surgical intervention for the RRP, as needed, throughout the course of the study. Surgery consisted of carbon dioxide laser ablation or microresection of the papilloma with a laryngeal skimmer blade and the Magnum powered hand instrument (Medtronics Xomed, Jacksonville, Florida). The choice between the two ablative techniques was determined by the surgeon's preferred technique. All of the adults and half of the children received carbon dioxide laser ablation. The difference in treatment methods between pediatric subjects was due to there being two different treating surgeons. The individual treating surgeon assessed the symptoms of the subjects and determined the time interval between treatments. All surgeries were documented via videoendoscopy. While the patient was under anesthesia, the surgeon ablated the papilloma by either carbon dioxide laser or microresector and then injected active drug or placebo into the tumor base.

With regard to cidofovir concentration, the FDA initially allowed a concentration of $0.3 \mathrm{mg} / \mathrm{mL}$ for children (less than 18 years of age) and $0.75 \mathrm{mg} / \mathrm{mL}$ for adults (18 years of age and older). Midway into the study, the FDA permitted an increase to $5 \mathrm{mg} /$ $\mathrm{mL}$ for both children and adults. The number of injections per treatment and the volume of each injection were at the discretion of the surgeon. No more than 6 treatments were performed per patient within
TABLE 1. PARTICIPANT CHARACTERISTICS, DRUG CONCENTRATIONS, AND BASELINE RRP SEVERITY

\begin{tabular}{|c|c|c|c|c|}
\hline Study Group & $\begin{array}{c}\text { Age at } \\
\text { Baseline } \\
\text { (y) }\end{array}$ & Sex & $\begin{array}{l}\text { Cidofovir } \\
\text { Concentration } \\
(\mathrm{mg} / \mathrm{mL})\end{array}$ & $\begin{array}{c}\text { Derkay RRP } \\
\text { Severity } \\
\text { Score } \\
\text { (Baseline) }\end{array}$ \\
\hline \multirow[t]{10}{*}{ Cidofovir group } & 34 & $\mathrm{~F}$ & 0.75 & 13 \\
\hline & 48 & M & 0.75 & 33 \\
\hline & 42 & $\mathrm{~F}$ & 0.75 & 19 \\
\hline & 5 & M & 0.3 & 14 \\
\hline & 58 & M & 0.75 & 8 \\
\hline & 51 & M & 0.75 & 7 \\
\hline & 32 & M & 0.75 & 5 \\
\hline & 6 & M & 5.0 & 14 \\
\hline & 30 & M & 5.0 & 5 \\
\hline & 27 & M & 5.0 & 14 \\
\hline \multirow[t]{9}{*}{ Placebo group } & 9 & M & & 8 \\
\hline & 16 & $\mathrm{~F}$ & & 11 \\
\hline & 31 & M & & 20 \\
\hline & 41 & M & & 16 \\
\hline & 34 & M & & 23 \\
\hline & 39 & M & & 5 \\
\hline & 40 & M & & 9 \\
\hline & 15 & M & & 8 \\
\hline & 33 & M & & 8 \\
\hline \multicolumn{5}{|c|}{ RRP - recurrent respiratory papillomatosis. } \\
\hline
\end{tabular}

the 12-month time interval of the study.

Participant characteristics, drug concentrations, and baseline RRP severity are shown in Table 1. It can be noted that the study sample was predominantly male. Within the cidofovir treatment group, 6 of the 10 participants who received active drug had injections of $0.75 \mathrm{mg} / \mathrm{mL}, 1$ participant received 0.3 $\mathrm{mg} / \mathrm{mL}$, and 3 participants received $5 \mathrm{mg} / \mathrm{mL}$. The placebo group contained 9 participants.

Before inclusion in this study, and 48 hours before each cidofovir injection, laboratory tests confirmed that the (female) participants were not pregnant and that laboratory findings were not exclusionary for study participation. In addition, participants received telephone calls from a study nurse 1 and 3 days after surgery, and returned to the clinic for additional laboratory tests and laryngeal videoendoscopy 14 days after surgery to rule out potential medication toxicities.

Statistical Methods. The number of procedures performed over the 12-month follow-up period and the 2-month and 12-month improvements in Derkay Severity Score, Voice Handicap Index (VHI) score, and Health-Related Quality of Life (HR-QOL) score were compared between the cidofovir and placebo groups with a Wilcoxon rank sum test. The withingroup improvement at 2 months and 12 months was examined with a Wilcoxon rank sum test. We considered $\mathrm{p}$ values of less than .05 to be significant. 
TABLE 2. DERKAY SEVERITY SCORE BEFORE TREATMENT AND AT 2 AND 12 MONTHS DURING TREATMENT OF RRP WITH CIDOFOVIR OR PLACEBO

\begin{tabular}{|c|c|c|c|c|c|c|c|c|}
\hline \multirow[b]{2}{*}{ Study Group } & \multicolumn{2}{|c|}{ Age $(y)$} & \multicolumn{2}{|c|}{ Before Injection } & \multicolumn{2}{|c|}{ At 2 mo } & \multicolumn{2}{|c|}{ At $12 \mathrm{mo}$} \\
\hline & Average & Range & Average & Range & Average & Range & Average & Range \\
\hline Cidofovir group $(\mathrm{n}=10)$ & 32 & $5-53$ & 13.2 & $5-33$ & $3.7^{*}$ & $0-11$ & $2.7^{*}$ & $0-13$ \\
\hline $\begin{array}{l}\text { Placebo group }(\mathrm{n}=9) \\
{ }^{*} \mathrm{p}<.05\end{array}$ & 29 & $10-40$ & 12 & $5-23$ & $3.6^{*}$ & $1-11$ & $5.1^{*}$ & $0-16$ \\
\hline
\end{tabular}

All analyses were performed with SAS statistical software version 9.1, SAS Institute Inc (Cary, North Carolina).

\section{RESULTS}

There were a total of 19 patients enrolled in the study. Ten were randomized to the cidofovir group, and 9 into the placebo group. The average age in the cidofovir group was 32 years (range, 5 to 58 years). There were 2 female and 8 male patients in this group. The concentrations for cidofovir injected ranged from 0.3 to $5.0 \mathrm{mg} / \mathrm{mL}$, and the volumes injected ranged from 0.5 to $3 \mathrm{~mL}$. There were no significant differences seen in the volumes injected into the placebo group and the treatment group. The amount injected was determined by the treating surgeon and was based on the extent of disease and the size of the larynx. There was 1 participant in the $0.3-\mathrm{mg} / \mathrm{mL}$ group, there were 6 participants in the $0.75-\mathrm{mg} / \mathrm{mL}$ group, and there were 3 participants in the $5-\mathrm{mg} / \mathrm{mL}$ group. The placebo group had an average age of 28 years (range, 9 to 40 years). There were 8 male participants and 1 female participant in this group.

The average starting Derkay Severity Scores were 13.2 (range, 5 to 33 ) for the cidofovir group and 12 (range, 5 to 23) for the placebo group. The average numbers of procedures during the 12-month study were 2.9 for the cidofovir group and 3 for the pla-

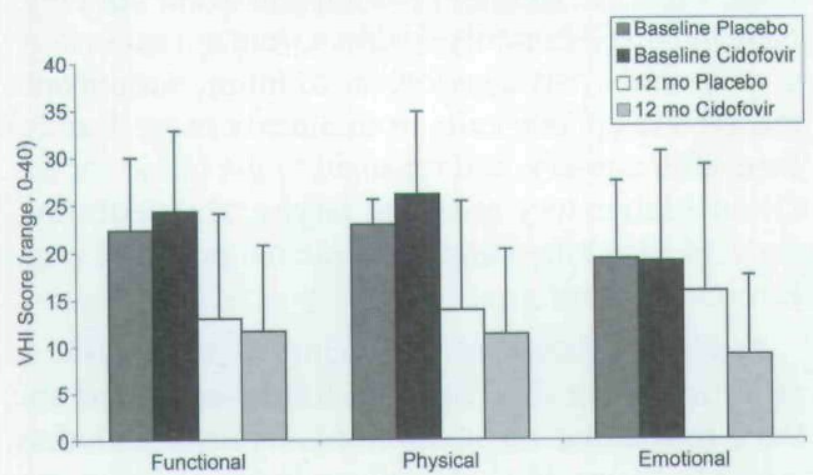

Fig 1. Voice Handicap Index (VHI) scores for placebo and cidofovir groups at baseline and at 12-month interval. There was significant decrease in VHI score after treatment of recurrent respiratory papillomatosis in both placebo and cidofovir groups. There was no difference seen between cidofovir and placebo groups. Lower VHI score correlates with better perceived voice-related quality of life. cebo group. There were no statistically significant differences between the two groups for these parameters.

As seen in Table 2, there were statistically significant decreases in the Derkay Severity Score seen at 2 months and at 12 months. Specifically, these decreased scores were 3.7 points (range, 0 to 11 ) and 2.7 points (range, 0 to 13 ), respectively, in the cidofovir group. These decreased scores were also demonstrated in the placebo group: 3.6 (range, 1 to 11) at 2 months and 5.1 (range, 0 to 16 ) at 12 months. Although there was a statistical difference between the initial Derkay Severity Score and the scores obtained at 2 and 12 months for the cidofovir $(\mathrm{p}<.05)$ and placebo $(\mathrm{p}<$ .05 ) groups, there was not a statistically significant difference between the cidofovir and placebo groups at any time point. Although there was a trend to a lower Derkay Severity Score in the cidofovir group at 12 months compared to the placebo group, the difference was not statistically significant.

As shown in Fig 1, a statistically significant improvement in the functional subset of the VHI score was observed in both the placebo and cidofovir groups $(\mathrm{p}<.05)$ after 12 months of participation in the study, although there were no differences between the 2 groups. There was also a significant improvement in the Total VHI score and the VHI subset (emotional and physical) scores in the cidofovir group $(\mathrm{p}<.05$; Fig 2$)$. Although there was a trend toward improved scores for the emotional and phys-

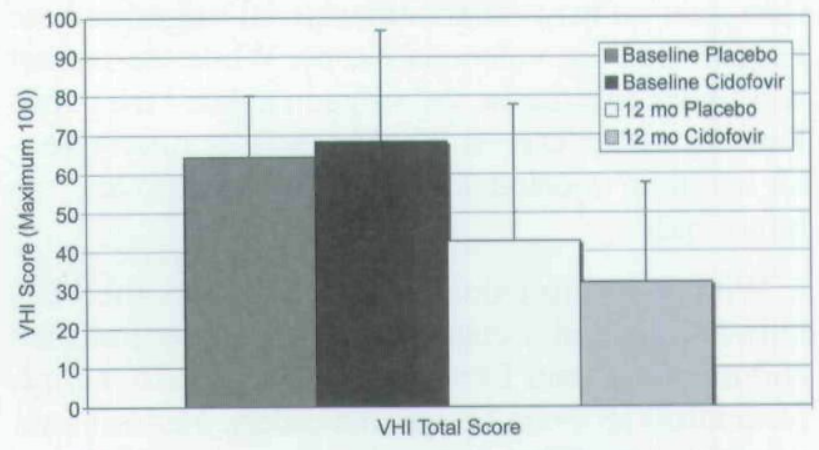

Fig 2. VHI total score for cidofovir and placebo groups at baseline and at 12 months. Maximum score is 100 . Lower score indicates improved perceived voice-related quality of life. Although there is trend toward improvement in both placebo and cidofovir groups, only cidofovir group reached statistical significance $(\mathrm{p}<.007)$. 


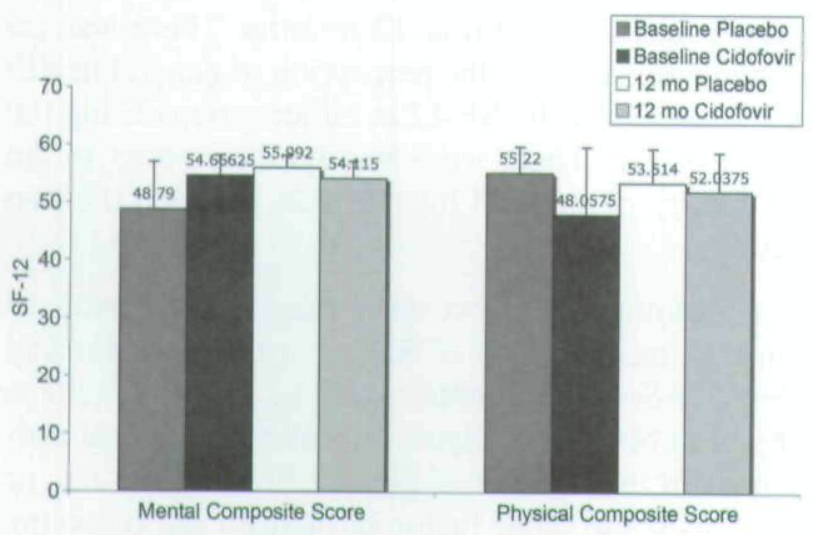

Fig 3. Short Form 12 (SF-12) scores at baseline and 12 months after treatment for cidofovir and placebo groups. Normal average mean is 50 (SD, 10). Lower score indicates poorer perception of general health quality. Two components of SF-12 are Mental Composite Score and Physical Composite Score. There were no statistical differences seen within groups at different time points or between placebo and cidofovir groups.

ical components and for the Total VHI score in the placebo group, they did not reach statistical significance. The HR-QOL (SF-12; Fig 3) and the PedsQL did not demonstrate differences from preinjection to posttreatment in either group. We saw no study-related adverse reactions to the injections in either the placebo group or the cidofovir group.

\section{DISCUSSION}

Our study showed a significant decrease in the severity of RRP seen at 2 and 12 months after intralesional injection with cidofovir at the time of surgical removal of gross disease. However, this significant difference was also seen in the placebo group. We saw a dramatic decrease in the Derkay Severity Score and improvement in the VHI after intralesional injection of either cidofovir or placebo. The cidofovir group trended toward a better Derkay Severity Score 12 months after treatment than did the placebo group, but the difference did not reach statistical significance. It is possible that the low concentration of drug used and the interval between treatments did not allow for significant differences to be seen. The cidofovir group scored better on the Total VHI than did the placebo group.

As surgical microresection is currently the standard of care, we used the cidofovir injections as an adjuvant to surgical ablation. We allowed the surgi$\mathrm{cal}$ indications for the management of RRP to dictate the frequency of cidofovir injection. Some case series have utilized frequent intralesional injections for therapy. They have escalated the intralesional injections to every other week for several injections. ${ }^{19}$ Some investigators have reported a better response to treatment with higher dosages and increased fre- quency in adults in uncontrolled trials. ${ }^{2}$ Although our treatment protocol differed in the frequency of injections from these other studies, we too showed a decrease in the severity of RRP with treatment.

An important difference of this study from others is the addition of a placebo control group. This placebo group had intralesional injections of saline solution. The surgeon and subject were blinded as to whether the injectate was placebo or drug. We added this group to decrease bias. No other study regarding the treatment of RRP with cidofovir has included a placebo group. The recognized but unpredictable behavior of spontaneous remission and reactivation confounds the study of treatment efficacy in RRP patients. The presence of spontaneous changes in disease expression requires that researchers control for this possibility in their study design. Wellmeaning but inherently biased observations may also subvert reported outcomes if the observers are not blinded to treatment.

Although we were able to standardize treatment in our placebo and drug groups during the study, the treatment of all of our subjects prior to the study could not be controlled, because some of these patients received treatment before being referred to our institution for management. The difference between their prior treatment and the overall approach to treatment during this study may account for some of the parallel improvements seen in both the test and control groups.

There were also fewer patients in the pediatric group, in part because there are fewer pediatric patients than adults with papillomatosis in our community. Recruitment issues may have also played a role in the pediatric population, as parents may be less willing to enroll their children in placebo-controlled studies rather than open treatment studies. The sparse pediatric population in this study may make it difficult to compare our findings with those studies whose primary population was pediatric. Comparison of our findings to those of studies of RRP in children is further limited by the possibility that there may be some metabolic or immunologic differences between the responses of children and adults to treatment with cidofovir for RRP.

The antiviral cidofovir has been used intralesionally with various frequencies of administration and in various concentrations. The typically reported injection concentration has been between 2.5 and 10 $\mathrm{mg} / \mathrm{mL} .{ }^{20}$ The FDA restricted the concentration of cidofovir we could use. We were required to use much lower concentrations than those typically reported in the literature. This, in part, was due to possible toxicity seen with cidofovir utilization. We, however, ob- 
served no adverse effect from the administration of cidofovir. During the study, the FDA allowed us to increase our drug concentration. Although the number of subjects in the higher drug concentration group was small, no differences were seen between the low and high concentration groups. We thought that increasing the concentration of the drug in the middle of the study would not adversely affect the results. We did not want to discard the results from the patients with lower concentrations, as they had already finished the protocol. We also felt that at worst, the increase would dilute the change we would see. $\mathrm{Al}$ though improvements were seen in the Derkay Severity Score and the VHI at 12 months in the cidofovir group, it is possible that statistical significance was not reached as compared to the placebo group because of the initial low drug concentration.

Along with the restriction of the drug dosage as required by the FDA, our study had a relatively small sample size. Even with a small sample size, however, the data reached statistical significance during analysis. As did other studies, ours showed a dramatic decrease in severity score of the RRP with cidofovir treatment - a finding that also characterized our placebo group.

This study showed a statistically significant improvement in Derkay Severity Score in patients treated with adjuvant cidofovir for RRP at 2 and 12 months after initiation of therapy. This improvement, however, was also seen in our placebo group. The VHI scores also significantly improved at 12 months for the cidofovir group. This finding dif- fers from the placebo at 12 months. There was no change, however, in the perception of general health as measured by the SF-12 in either group during the study period. There were also no differences in the frequency of surgical intervention between the two groups.

Cidofovir or placebo, when used as an adjuvant to surgical management of RRP, improves the Derkay Severity Score at 2 months and 12 months. Perhaps the placebo effect helped improve the clinical outcomes of these patients' Derkay Severity Scores, or perhaps some other factor influenced the outcome, such as intralesional hydrostatic dissection. As in many other case series, we found an improvement in clinical severity with intralesional cidofovir. Our placebo group, however, did just as well. Accordingly, we are unable to provide proof of efficacy of cidofovir in the treatment of RRP at the dosage and frequency of administration employed in this study.

Our data provide justification for further studies to determine the efficacy of cidofovir in treating respiratory papillomatosis. Our initial study design was to include multiple institutions. We were unable, however, to recruit other institutions to join our study, mainly because of the low initial dose of cidofovir we were required to use. We believe that a multi-institutional trial will help to increase the power of the study by increasing the number of subjects. We also believe that a placebo group is important in the study design. Had we not included a placebo group, we, too, would have attributed the improvement in clinical outcome to the cidofovir alone.

\footnotetext{
Acknowledgments: The authors gratefully acknowledge the following people and organizations who were instrumental in the completion of this work: Gilead Sciences (for the donation of the medication), the University of Wisconsin Pharmaceutical Research Center, the University of Wisconsin Medical School General Clinical Research Center, the University of Wisconsin Department of Surgery, Dr Diane Heatley, Dr Gregory Sewall, Dr Glen Leverson, Stacy Cohen, Edith Swift, Amy Kramer, Eva van Leer, and Jessica Tiede.
}

\section{REFERENCES}

1. Wiatrak BJ, Wiatrak DW, Broker TR, Lewis L. Recurrent respiratory papillomatosis: a longitudinal study comparing severity associated with human papilloma viral types 6 and 11 and other risk factors in a large pediatric population. Laryngoscope 2004;114(suppl 104):1-23.

2. Naiman AN, Ceruse P, Coulombeau B, Froehlich P. Intralesional cidofovir and surgical excision for laryngeal papillomatosis. Laryngoscope 2003;113:2174-81.

3. Naiman AN, Abedipour D, Ayari S, et al. Natural history of adult-onset laryngeal papillomatosis following multiple cidofovir injections. Ann Otol Rhinol Laryngol 2006;115:175-81.

4. Derkay CS. Task force on recurrent respiratory papillomas. A preliminary report. Arch Otolaryngol Head Neck Surg 1995;121:1386-91.

5. Clifford GM, Gallus S, Herrero R, et al. Worldwide distribution of human papillomavirus types in cytologically normal women in the International Agency for Research on Cancer HPV prevalence surveys: a pooled analysis. Lancet 2005;366:991-8.
6. Sisk J, Schweinfurth JM, Wang XT, Chong K. Presence of human papillomavirus DNA in tonsillectomy specimens. Laryngoscope 2006;116:1372-4.

7. Schraff S, Derkay CS, Burke B, Lawson L. American Society of Pediatric Otolaryngology members' experience with recurrent respiratory papillomatosis and the use of adjuvant therapy. Arch Otolaryngol Head Neck Surg 2004;130:1039-42.

8. Myer CM III, Willging JP, McMurray S, Cotton RT. Use of a laryngeal micro resector system. Laryngoscope 1999;109: 1165-6.

9. Lee AS, Rosen CA. Efficacy of cidofovir injection for the treatment of recurrent respiratory papillomatosis. J Voice 2004;18:551-6.

10. Mandell DL, Arjmand EM, Kay DJ, Casselbrant ML, Rosen CA. Intralesional cidofovir for pediatric recurrent respiratory papillomatosis. Arch Otolaryngol Head Neck Surg 2004; 130:1319-23.

11. Pransky SM, Albright JT, Magit AE. Long-term follow- 
up of pediatric recurrent respiratory papillomatosis managed with intralesional cidofovir. Laryngoscope 2003;113:1583-7.

12. Pransky SM, Brewster DF, Magit AE, Kearns DB. Clinical update on 10 children treated with intralesional cidofovir injections for severe recurrent respiratory papillomatosis. Arch Otolaryngol Head Neck Surg 2000;126:1239-43.

13. Pransky SM, Magit AE, Kearns DB, Kang DR, Duncan NO. Intralesional cidofovir for recurrent respiratory papillomatosis in children. Arch Otolaryngol Head Neck Surg 1999;125: 1143-8.

14. Snoeck R, Wellens W, Desloovere C, et al. Treatment of severe laryngeal papillomatosis with intralesional injections of cidofovir [(S)-1-(3-hydroxy-2-phosphonylmethoxypropyl)cytosine]. J Med Virol 1998;54:219-25.

15. Woo P. Office-based laryngeal procedures. Otolaryngol Clin North Am 2006;39:111-33.

16. Derkay CS, Malis DJ, Zalzal G, Wiatrak BJ, Kashima
HK, Coltrera MD. A staging system for assessing severity of disease and response to therapy in recurrent respiratory papillomatosis. Laryngoscope 1998;108:935-7.

17. Ware J Jr, Kosinski M, Keller SD. A 12-Item Short-Form Health Survey: construction of scales and preliminary tests of reliability and validity. Med Care 1996;34:220-33.

18. Varni JW, Seid M, Rode CA. The PedsQL: measurement model for the pediatric quality of life inventory. Med Care 1999; 37:126-39.

19. Chhetri DK, Shapiro NL. A scheduled protocol for the treatment of juvenile recurrent respiratory papillomatosis with intralesional cidofovir. Arch Otolaryngol Head Neck Surg 2003; 129:1081-5.

20. Akst LM, Lee W, Discolo C, Knott D, Younes A, Koltai PJ. Stepped-dose protocol of cidofovir therapy in recurrent respiratory papillomatosis in children. Arch Otolaryngol Head Neck Surg 2003;129:841-6. 
Copyright of Annals of Otology, Rhinology \& Laryngology is the property of Annals Publishing Company and its content may not be copied or emailed to multiple sites or posted to a listserv without the copyright holder's express written permission. However, users may print, download, or email articles for individual use. 